\title{
COMPARATIVE EVALUATION OF EFFECTIVENESS OF CASEIN PHOSPHOPEPTIDE AMORPHOROUS CALCIUM PHOSPHATE AND SODIUM FLOURIDE VARNISH IN DENTINE HYPERSENTIVITY
}

\author{
Beenish Abbas, Madeeha Sattar, Sana Abbas*, Shoaib Rahim, Qamar Ishfaque**, Mehreen Wajahat*** \\ Foundation University College of Dentistry, Islamabad Pakistan, *Armed Forces Institute of Ophthalmology/National University of Medical Sciences (NUMS) \\ Rawalpindi Pakistan, ${ }^{* *}$ Armed Forces Institute of Dentistry/National University of Medical Sciences (NUMS) Rawalpindi Pakistan, ${ }^{* * *}$ Avicenna Medical and \\ Dental College, Lahore Pakistan
}

\section{ABSTRACT}

Objective: To determine efficacy of casein phosphopeptide amorphous calcium phosphate and sodium fluoride varnish in managing dentine hypersentivity.

Study Design: Quasi-experimental study.

Place and Duration of Study: Foundation University College of Dentistry, Islamabad, from Jun to Nov 2020.

Methodology: Patients of both gender 20-60 years of age with ASA “American Society of Anesthesiologists physical status classification" status I (completely healthy fit patient) or status II (patient has mild systemic disease) diagnosed with dentine hypersensitivity of incisors, canines, premolars with clinical evidence of erosion, abrasion, gingival recession not requiring restorative treatment were enrolled in the study. At first visit baseline, dentine hypersentivity was recorded by means of tactile and evaporative stimuli, response was quantified using visual analogue scale. Patients were randomly divided in two groups 1-casein phosphopeptide amorphous calcium phosphate, 2-sodium fluoride varnish desensitizing agent was applied conferring to manufacturer instructions. Visual analogue scale readings were assessed at base line and post treatment values was recorded at $7^{\text {th }}, 15^{\text {th }}, 30^{\text {th }}$ day.

Results: Total 80 patients enrolled in the study with a mean age of 36.26 \pm 8.91 years and age-range of 21-60 years. Visual analogue scale results of group- $1(\mathrm{n}=40)$ were recorded at baseline as $5.9 \pm 0.94$, 7th day $1.9 \pm 0.92,15^{\text {th }}$ day $1.4 \pm 0.81$ and 30th day 1.0 \pm 0.71. In case of group-2 Visual Analogue Score recorded to be $6.2 \pm 1.24,3.2 \pm 0.81,3.0 \pm 0.52$ and $2.7 \pm 1.12$ at baseline, $7^{\text {th }}, 15^{\text {th }}$, and $30^{\text {th }}$ day respectively.

Conclusion: Casein phosphopeptide amorphous calcium phosphate is more effective than sodium fluoride varnish in controlling pain associated with dentine hypersentivity.

Keywords: Casein phosphopeptide amorphous calcium phosphate, Dentine hypersentivity, Sodium fluoride varnish.

How to Cite This Article: Abbas B, Sattar M, Abbas S, Rahim S, Ishfaque Q, Wajahat M. Comparative Evaluation of Effectiveness of Casein Phosphopeptide Amorphorous Calcium Phosphate and Sodium Flouride Varnish in Dentine Hypersentivity. Pak Armed Forces Med J 2021; 71(5): 1656-1660. doi: https://doi.org/10.51253/pafmj.v71i5.5206

This is an Open Access article distributed under the terms of the Creative Commons Attribution License (https://creativecommons.org/licenses/by-nc/4.0/), which permits unrestricted use, distribution, and reproduction in any medium, provided the original work is properly cited.

\section{INTRODUCTION}

Dentin hypersentivity (DHS) is defined as a pain of short duration, sharp in character arising because of exposed dentine, in response to stimuli like tactile, evaporative and thermal. ${ }^{1}$ Hydrodynamic theory for DHS states that pain results from alteration in fluid movement in response to stimuli mentioned above resulting in activation of nociceptors at pulp dentine interface. Cunha-Cruz et al reported in a recent review that the prevalence of dentinal hypersentivity was $12.3 \% .^{2}$

Most frequently effected teeth are first premolars and canines, with molars being least affected. Peak prevalence is in age ranges between 30-39 years and the majority of cases are reported in individuals between 20-50 years. Combination of abrasive and erosive

Correspondence: Dr Qamar Ishfaque, Department of Dentistry, Armed Forces Institute of Ophthalmology, Rawalpindi Pakistan Received: 30 Aug 2020; revision received: 03 Feb 2021; accepted: 15 Feb 2021 tooth wear is thought to be responsible for causing DHS. Most commonly effected sites are buccal cervical regions. ${ }^{3}$ For screening prevention and monitoring of dentinal hypersentivity in general dental practice pragmatic guidelines must be followed, that can help in successful management of such cases. Diagnosis of DHS is quiet challenging, it is important to recognize that it is essentially a diagnosis of exclusion. There are vast array of commercially available in-office and over the counter products available claiming to be effective in pain associated with DHS. ${ }^{4}$ Investigators have reported that DHS has an association with tooth wear pre-dominantly, however a number of etiological factors predispose to DHS these includes abrasion, abfractive wedge shaped lesions, periodontal disease, gingival recession, periodontal surgical procedures and thin gingival biotype. ${ }^{3}$

There are two specific biological processes involved, firstly-there is lesion initiation because of dentine 
exposure due to loss of enamel and associated gingival recession. Secondly, once there is dentine exposure dentinal tubules are patent and exposed to oral environment. Mechanoreceptors are activated in response to minute fluid movement within the patent dentinal tubules. ${ }^{5}$ Clinical symptoms of DHS are quite variable in different patients, diagnosis is challenging due to subjective nature of pain. It is important to exclude any confounding factors from other orofacial pain conditions. Formulation of clear diagnosis based on sound clinical judgment is critical for successful management of clinical cases. Thorough medical and dental history must be recorded, detailed intraoral and extraoral examination is done before reaching a diagnosis. Site, onset, character, radiation of pain and associated symptoms are taken into account to formulate a treatment plan. ${ }^{6}$

An air blast using triple syringe or tactile stimulation using an explorer probe, together with a degree of discomfort reported by patient resulting from application of stimulus during clinical examination are acceptable to identify susceptible sites. Use of simple numeric scale score visual analogue scale is used in clinical practice to record severity of pain associated with dentine hypersentivity at baseline and to evaluate improvement over subsequent visits, this method gives pain score objectively. ${ }^{7}$ Dental professionals have a variety of treatment options available ranging from desensitizing agents like potassium nitrate based tooth paste as well as tubule blocking agents like strontium chloride/ oxalate compounds. ${ }^{8}$ Remineralizing agents like sodium fluoride varnish and casein phosphopeptide amorphous calcium phosphate are novel therapeutic agents available for management of DHS. ${ }^{9}$

Casein phosphopeptide (CPP) is a milk derived phosphoprotein, it has the ability to bind and stabilize soluble amorphous calcium phosphate (ACP) and it provide reservoir of calcium and phosphate ions on demineralized tooth surface. In the presence of saliva, ACP dissociates into calcium and phosphate, maintaining mineral supersaturation and promoting remineralization by formation of hydroxyapatite (HA) crystals. 10 The rationale of this study was to determine the effective therapeutic modality in management of DHS.

\section{METHODOLOGY}

This quasi-experimental study was carried out at Teaching Dental Institute from June to November 2020. The approval was taken from ethics research committee of the institute (ref no. AFID/R\&D-5/25-2020). The sample size of 80 subjects (40 in each group) was calculated by openEpi. Patients were enrolled in the study with two-sided confidence level(1-alpha) $95 \%$, $80 \%$ power of detection and equal proportion of case to controls. Hypothetical proportion of controls with exposure (40) and hypothetical proportion of cases with exposure (12.3) with odds ratio (0.21) as elaborated by Cunha et al. ${ }^{2}$ With consecutive non-probability sampling a total of 80 patients were enrolled. Group 1-casein phosphopeptide amorphous calcium phosphate (CPP ACP) $(n=40)$ and group 2-sodium fluoride varnish $(n=40)$.

Patients were interviewed, briefed, counselled about the procedure, and informed written consent was taken. At first appointments, a detailed history, extraoral, intraoral examination was carried out in all patients with necessary monitory of patient vital signs. Evaluation parameters was such that we could to adhere with our inclusion and exclusion criteria, was done to ensure patient safety, which is an utmost concern in dental treatment. Careful screening of the patients for fever and symptoms consistent with COVID19 was performed. Strict cross infection protocol was being followed.

Inclusion Criteria: Patient diagnosed with dentine hyper-sentivity from both genders with an age range between 20-60 years, American Society of Anesthesiologist's (ASA) status I, or II were enrolled.

Exclusion Criteria: Non-consenting patients, mentally handicapped, pregnant and lactating females, patients with moderate-severe cardiovascular or ischemic diseases, peri-odontal disease, active root caries, internal root resorption, tongue piercing, history of bleaching treatment in last 8 weeks were excluded from the study.

At first visit, pain associated with dentine hypersentivity was recorded by means of tactile and evaporative stimuli as baseline record. Evaporative stimuli were applied using triple syringe delivering air blast held perpendicular and $1 \mathrm{~cm}$ away while shielding adjacent teeth with rubber dam isolation. Two consecutive readings were recorded at $5 \mathrm{~min}$ interval. To confirm diagnosis further tactile stimulation using periodontal probe passed perpendicular to tooth surface with apical sweeps with initial pressure of $10 \mathrm{~g}$ and then gradually increased in increments of $10 \mathrm{~g}$ until subject responds. Pain response was quantified using visual analog scale (VAS). Patient was provided VAS to place marking on a line of $10 \mathrm{~cm}$ length. On this line, 0 was no pain and 10 very severe pain. Subjects were randomly allocated in 02 groups, group 1 with casein 
phosphopeptide amorphous calcium phosphate (CPP$\mathrm{ACP}$ ), group 2 with sodium fluoride varnish. Teeth were air dried before the application of desensitizing agents. Desensitizing agent was applied over the tooth surface under rubber dam application conferring to manufacturer's instruction and left undisturbed over the tooth for $60 \mathrm{~s}$. Patients were advised not to rinse eat drink for $30 \mathrm{mins}$ after appointment. Pretreatment assessment was done at baseline and post-treatment was done on 7 th, 15th and 30th day.

Data entered and analyzed using Statistical Package for Social Sciences (SPSS) version 22. Quantitative variables including age presented as mean and standard deviation. Qualitative variables including gender, ASA status presented in terms of frequency and percentage. Independent sample t-test was employed. The $p$-value of $\leq 0.05$ was considered statistically significant.

\section{RESULTS}

Total 80 patients enrolled in the study with a mean age of $36.26 \pm 8.91$ years and age-range of 21-60 with gender distribution of male $55(67.1 \%)$ and female $25(30.5 \%)$. Sixty nine (84.1\%) patients had American Society of Anesthesiologists (ASA) Status I whereas 11 (13.4\%) were from ASA Status II (Table-II). VAS results of group $1 \quad(n=40)$ were recorded at baseline as $5.9 \pm 0.94,7^{\text {th }}$ day $1.9 \pm 0.92,15^{\text {th }}$ day $1.4 \pm 0.81$ and $30^{\text {th }}$ day $1.0 \pm 0.71$. In case of group 2 Visual Analogue Score recorded to be $6.2 \pm 1.24,3.2 \pm 0.81,3.0 \pm 0.52$ and $2.7 \pm 1.12$ at baseline, $7^{\text {th }}, 15^{\text {th }}$, and $30^{\text {th }}$ day respectively (Table-II).

Table-I: Distribution of demographic profile.

\begin{tabular}{l|c|c}
\hline \multicolumn{2}{l|}{ Parameters } & n (\%) \\
\hline American Society of & I & $69(84.1 \%)$ \\
\cline { 2 - 3 } Anesthesiologists & II & $11(13.4 \%)$ \\
\hline \multirow{2}{*}{ Gender } & Male & $55(67.1 \%)$ \\
\cline { 2 - 3 } & Female & $25(30.5 \%)$ \\
\hline
\end{tabular}

Table-II: Comparison of visual analog scale scores at baseline $7^{\text {th }}, 15^{\text {th }}, 30^{\text {th }}$ day.

\begin{tabular}{l|c|c|c}
\hline Parameters & $\begin{array}{c}\text { Group 1 } \\
(\mathbf{n}=40) \\
\text { Mean } \pm \text { SD }\end{array}$ & $\begin{array}{c}\text { Group 2 } \\
(\mathbf{n}=40) \\
\text { Mean } \pm \text { SD }\end{array}$ & $\begin{array}{c}p- \\
\text { value }\end{array}$ \\
\hline $\begin{array}{l}\text { Baseline VAS (Visual } \\
\text { Analog Scale) score }\end{array}$ & $5.9 \pm 0.94$ & $6.2 \pm 1.24$ & $0.02 *$ \\
\hline $\begin{array}{l}\text { 7th day VAS (Visual } \\
\text { Analog Scale) score }\end{array}$ & $1.9 \pm 0.92$ & $3.2 \pm 0.81$ & 0.70 \\
\hline $\begin{array}{l}\text { 15th day VAS (Visual } \\
\text { Analog Scale) score }\end{array}$ & $1.4 \pm 0.81$ & $3.0 \pm .52$ & $0.001^{*}$ \\
\hline $\begin{array}{l}\text { 30th day VAS (Visual } \\
\text { Analog Scale) score }\end{array}$ & $1.0 \pm 0.71$ & $2.7 \pm 1.12$ & $0.001^{*}$ \\
\hline $\begin{array}{l}\text { *Significant p-value; p-value was calculated by applying } \\
\text { Independent sample t-test }\end{array}$
\end{tabular}

\section{DISCUSSION}

Data analysis of research interpreted statistically significant efficacy of CPP-ACP in reducing pain associated with DHS as expressed by VAS scores at $15^{\text {th }}$ day and $30^{\text {th }}$ day, due to its potential for inducing remineralization of dentinal tubules. Ortiz et al, conducted a randomized controlled trial to determine efficacy of casein phosphopeptide-amorphous calcium phosphate fluoride (CPP-ACPF) and photo bio modulation (PBM) in the management of DHS, and effect of this management line on the health-related quality of life (HRQL). There inter-group comparison with thermal and evaporative stimuli presented that $\mathrm{CPP}-\mathrm{ACPF}+\mathrm{PBM}$ significantly reduced DHS when this treatment modality was compared with the rest of treatment options, after one-month follow-up. CPP-ACPF+PBM group showed statistically significant efficacy with a $p$-value $(p<0.05)$. A positive impact on the HRQL due to reduction in pain associated with dentin hypersensitivity is reported with treatment by CPP-ACPF with PBM. ${ }^{11}$

Fernando et al conducted an in vitro study on remineralizing potential of CPP-ACP + stannous fluoride $(\mathrm{SnF} 2)$ solution, significantly higher enamel remineralization was observed in vitro by the combined CPP$\mathrm{ACP}+\mathrm{SnF} 2$ solution compared with the CPP-ACP + $\mathrm{NaF}$ solution. The extent of remineralization with the $\mathrm{CPP}-\mathrm{ACP}+\mathrm{SnF} 2$ treatment was $32 \%$ greater than with the CPP-ACP + NaF treatment. CPP-ACP and SnF2 showed synergism, combination of SnF2 to CPP-ACP resulted in increased complex stability, and greater potential for ion delivery at the tooth surface this unique interaction of Sn F2 and CPP-ACP resulted in formation of Stannous ions aggregated CPP nanocomplexes, and elevated ion release and self-assembly of cross-linkage CPP nanofilaments upon contact with the tooth surface. They concluded that, the combination of CPP$\mathrm{ACP}$ and SnF2 might considerably improve their efficacy in prevention and treatment DHS, finding consistent with our study. A nano-coating formed on the tooth surface drastically improves DHS. ${ }^{12}$

Douglas et al, conducted a study to evaluate, that DHS treatments were able to improve individuals' oral HRQL. DHS was assessed by evaporative, cold, and tactile stimuli, as we did in our study. Oral HRQoL was evaluated by questionnaires. In-home and inoffice desensitizing agents like $\mathrm{CPP}-\mathrm{ACP}$, dentine bonding agent, potassium nitrate-based gels were used for DHS treatment. Their study reported statistically significant reduction of DHS and significant positive impact on oral HRQoL after treatment $(p<0.05)$. Studies indica- 
ted decreasing of DHS and improving of oral HRQoL after DHS treatment. ${ }^{13}$ Alleviation of pain associated with DHS improved oral HRQoL in our patient group.

Al-Shamrani et al, conducted research to investigate the effect of CPP-ACP, neutral sodium fluoride gel (Flor-Opal), and nano-carbonate Apatite Paste (nCAP), on enamel surface roughness and postoperative DHS. Their results revealed that after the application of the three surface desensitizing treatments, enamel treated with nano carbonate appetite paste showed a significant reduction in surface roughness $(p=0.002)$ when compared with other two therapeutic agents. Results did not show significant effectiveness of sodium fluoride varnish in reducing DHS. Conclusion of their study was nanocarbonate Apatite Paste was considerably more effective in reducing enamel surface roughness and associated hypersensitivity. ${ }^{14}$

Dawood et al, conducted a study on biocompatibility and remineralization potential of CPP-ACP. Their study concluded that CPP-ACP promote mineralization by increasing bioavailability of calcium and phosphate ions needed for remineralization thereby reducing DHS. Biocompatibility of CPP-ACP was proved in this study and alkaline phosphatase activity of the osteoblast-like cells was significantly increased ( $p$ $<0.05$ ) by CPP-ACP and CPP-ACFP. This material also enhanced formation of calcium mineral deposits, and the secretion of mineralization-related proteins (collagen type I and osteocalcin). ${ }^{15}$ Conclusion of this study supports our findings.

Tarique et al, did a comparative analysis on efficacy of three desensitizing agents i.e., $5 \%$ sodium fluoride $(\mathrm{NaF}), 5 \%$ potassium nitrate $(\mathrm{KNO} 3)$ and $\mathrm{CPP}-$ $\mathrm{ACP}$ in reducing DHS after in-office vital tooth bleaching. Results of their study was significant at $p$-value $<0.05$, thereby concluding CPP-ACP substantially reduces symptoms associated with DHS and the other two materials are having nearly the same efficacy but inferior to CPP-ACP. ${ }^{16}$ In their study sensitivity was checked on alternating days using VAS for up to one month mean was taken to evaluate the results.

Therefore, our treatment group and results were significant at $p$-value $<0.05$ showing better efficacy of CPP-ACP over sodium fluoride varnish. A paradigm shift is emerging in dentistry and dental treatments are now aimed at providing minimally invasive and remineralization management approaches thereby conserving tooth structure. ${ }^{17,18} \mathrm{CPP}-\mathrm{ACP}$ is emerging novel material there is scarcity of literature regarding preventive, remineralizing potential of this material we should be doing what is best available for our patients to improve their health related quality of life and to pace up with evolution in field of dentistry.

\section{CONCLUSION}

Casein phosphopeptide amorphous calcium phosphate (CPP-ACP) provides better control of symptoms when compared with sodium fluoride varnish, therefore it is an essential therapeutic modality in successful management of DHS cases.

\section{Conflict of Interest: None.}

\section{Authors' Contribution}

BA: Conception of idea, data collection, manuscript writing, MS: Conception of idea, data collection, SA: Data analysis, SR: Manuscript writing, QI: Data analysis, MW: Manuscript writing.

\section{REFERENCES}

1. Gillam DG. A new perspective on dentine hypersensitivityguidelines for general dental practice. Dent Update 2017; 44(1): 33-42.

2. Cunha-Cruz J, Wataha JC, Heaton LJ, Rothen M, Sobieraj M, Scott J, et al. The prevalence of dentin hypersensitivity in general dental practices in the northwest United States. J Am Dent Assoc 2013; 144(3): 288-296.

3. Lin YH, Gillam DG. The prevalence of root sensitivity following periodontal therapy: A systematic review. Int J Dent 2012; 2012(2): 407023.

4. Kopycka-Kedzierawski DT, Meyerowitz C, Litaker MS, Chonowski S, Heft MW, Gordan V V, et al. Management of Dentin Hypersensitivity by National Dental Practice-Based Research Network practitioners: Results from a questionnaire administered prior to initiation of a clinical study on this topic. BMC Oral Health 2017; 17(1): 41-45.

5. Olley RC, Moazzez R, Bartlett D. The relationship between incisal/occlusal wear, dentine hypersensitivity and time after the last acid exposure in vivo. J Dent 2015; 43(2): 248-52.

6. Gillam D, Chesters R, Attrill D, Brunton P, Slater M, Strand P, et al. Dentine hypersensitivity - Guidelines for the management of a common oral health problem. Dent Update 2013; 40(7): 514524.

7. Chałas R, Wójcik-Chęcińska I, Zamościńska J, Bachanek T. Assessment of pain intensity in patients with dentin hypersensitivity after application of prophylaxis paste based on calcium sodium phosphosilicate formula. Med Sci Monit 2015; 21(2): 2950-2955.

8. Karim BFA, Gillam DG. The efficacy of strontium and potassium toothpastes in treating dentine hypersensitivity: A systematic review. Int J Dent 2013; 2013(2): 573258.

9. Praveen R, Thakur S, Kirthiga M, Narmatha M. Comparative evaluation of a low-level laser and topical desensitizing agent for treating dentinal hypersensitivity: A randomized controlled trial. J Conserv Dent 2018; 21(5): 495-499.

10. Bou Chebel F, Zogheib CM, Baba NZ, Corbani KA. Clinical comparative evaluation of $\mathrm{Nd}$ :yag laser and a new varnish containing casein phosphopeptides-amorphous calcium phosphate for the treatment of dentin hypersensitivity: A prospective study. J Prosthodont 2018; 27(9): 860-867.

11. Ortiz MIG, de Melo Alencar C, de Paula BLF, Alves EB, Araújo JLN, Silva CM. Effect of the casein phosphopeptide-amorphous calcium phosphate fluoride (CPP-ACPF) and photobiomodula- 


\section{Dentine Hypersentivity}

tion (PBM) on dental hypersensitivity: A randomized controlled clinical trial. PLoS One 2019; 14(12): e0225501.

12. Fernando JR, Shen P, Sim CPC, Chen YY. Self-assembly of dental surface nanofilaments and remineralisation by SnF2 and CPPACP nanocomplexes. Sci Rep 2019; 9(1): 1285-1290.

13. Douglas-de-Oliveira DW, Vitor GP, Silveira JO, Martins CC, Costa FO, Cota LOM. Effect of dentin hypersensitivity treatment on oral health related quality of life-A systematic review and meta-analysis. J Dent 2018; 71(1): 1-8.

14. Al-shamrani A, Awliya W. The effect of neutral sodium fluoride, casein phosphopeptide-amorphous calcium phosphate, and nano-carbonate apatite on enamel surface roughness and staining after home bleaching. Int J Dent Oral Health 2019; 5(4): $1-6$.
15. Dawood AE, Manton DJ, Parashos P, Wong RH, Singleton W, Holden JA, et al. Biocompatibility and osteogenic/ calcification potential of casein phosphopeptide-amorphous calcium phosphate fluoride. J Endod 2018; 44(3): 452-457.

16. Tarique N, Awan R, Saleh MI. Vital tooth bleaching and management of post operative sensitivity: A clinical trial evaluating the efficacy of different desensitizing materials. Pak J Med Health Sci 2017; 11(4): 1564-1567.

17. Farooq I, Moheet IA. A review of novel dental caries preventive material: casein phosphopeptideamor-phous calcium phosphate complex. King Saud Univ J Dent Sci 2013; 4(2): 47-51.

18. Moogi P, Rupesh MC. A novel desensitizer paste containing calcium phosphate: Randomized, placebo-controlled, doubleblinded and 6 months trial. World J Dent 2017; 8(6): 440-444. 